\title{
NONLINEAR MODELLING AND SIMULATION OF INDUSTRIAL WET GRINDING PROCESS
}

\author{
V. S. S. Rameshkumar Junnuri, Kishalay Mitra and Jayesh Barve^ \\ Engineering Technology Development, Engineering and Industrial Services \\ Tata Consultancy Services Limited, Pune- 411048 INDIA \\ ^Corresponding Author: jayesh.b@tcs.com
}

\begin{abstract}
The multiple linear models based piece-wise linearization approach is used to obtain control-oriented non-linear model of the non-linear multivariable wet grinding process of an industrial lead-zinc ore beneficiation plant. The overall process outputs are computed as a weighted sum of outputs from multiple models identified at several operating zones. The output weights of linear models are computed by a pseudo fuzzy supervisor. The linear models are identified using a transfer matrix identification technique using standard $\mathrm{TF}$ structures, and genetic algorithm based constrained optimisation formulation for the parameter estimation. A rigorous, plant validated model based wet grinding process simulator is used to obtain the identification and validation data for the proposed approach. Copyright (C) 2005 IFAC
\end{abstract}

Keywords: Nonlinear Systems, Process Identification, Fuzzy Supervision, Industrial Production System, Process Control

\section{INTRODUCTION}

Grinding plays a critical role in most of the ore beneficiation operations in mineral processing plants. The size of the particles produced by the grinding operation also drives the performance of the downstream process units, e.g. flotation. Hence, the better process understanding and tighter control of the grinding process is of utmost interest to the mineral process engineers.

For better process understanding, the most efficient tool is process modelling and simulation. Over the years, the modelling of the grinding process has attained a reasonable state of accuracy and robustness (Herbst et al. (1973, 1983), Kinneberg et al. (1984), Lynch et al. (1975), Rajamani et al. (1984, 1991a)). Most of the cases, these approaches follow the hybrid path of physical and empirical routes where mass balance of the materials is carried out using physical laws and the separation kinetics are modelled using empirical methods. Such models usually require some specific process information such as process equipment design specifications, and the process parameters e.g. selection function, breakage function, and kinetic parameters etc. Mostly, such parameters are difficult to know exactly and accurately in industrial plants. Hence, it is required to have some specific tuning parameters in such rigorous models and tune and validate them using the plant data obtained by performing welldesigned but tedious plant tests. In general, such exercise of tuning the model parameters becomes quite cumbersome and computationally demanding, mainly because the parameters appear in the model in a quite complex mathematical form. Many researchers have worked on such model parameter tuning techniques with respect to wet grinding process. Some of them cover several aspects of single as well as multiple objective optimizations of industrial grinding operations (Birch et al. (1972), Bryson et al. (1969), Herbst et al. (1979), Lapidus et al. (1967), Rajamani et al. (1991b), Mitra and Gopinath (2004)). These kind of hybrid grinding models are reported to work really well in many cases (Mitra and Gopinath (2004)) if tuned properly with the plant using the data obtained from hardware sensors in at least some bare minimum flow streams.

For tighter control of the industrial wet grinding process in mineral industry, compared to the conventional control algorithms (e.g PID), the multivariable control algorithm such as Model Predictive Control (MPC) can be considered more appropriate, particularly for the supervisory control (Maciejowski, 2002). This is because the MPC can efficiently and systematically handle the issues like a) significant amount of interaction among various 
process/operational variables, and 2) the requirement to operate the process within the operational and equipment constraints, usual in an industrial wet grinding process. Additionally, MPC provides several other benefits (Maciejowski, 2002), (Morari and Lee, 1999).

The MPC algorithms inherently require the dynamic model of the process. Ideally, it is good if the first principle based rigorous models described above are used as the MPC model, because such models are quite accurate, and are applicable in a wider operating region. However, such rigorous models are not suitable for use in MPC, particularly for the online industrial control applications. Because such models - 1) are computationally very demanding, and 2) are mostly the non-linear models. Although, use of non-linear models are possible in Non-Linear Model Predictive Control (NLMPC), but the NLMPC algorithms based on non-linear models are still not suitable and popular for online industrial control applications. Because NLMPC require solving non-linear programming (optimisation) problem, for which the reliable and efficient online algorithms are still scarce. On the other hand, the MPC algorithms based on linear models are popular and proven in wide range of industrial applications.

Thus, the conflicting requirement of modelling the non-linear grinding process accurately, but without using non-linear models (Bequette et. Al. 2003), has motivated us to propose using multiple linear models based piece-wise linearization approach for modelling the non-linear grinding process in this paper. The paper is organized as follows. Section-2 describes the industrial wet grinding process of an industrial lead-zinc ore beneficiation plant, along with the brief description of the rigorous model based plant simulator. The rigorous simulator was developed, tuned, and validated well with the plant data in the earlier work by (Mitra and Gopinath, 2004), and is used as a plant emulator in this work. In section-3, the proposed multiple linear model based non-linear modelling approach is described. In Section-4 the results of the proposed approach are presented and discussed. Section- 5 contains the concluding remarks.

\section{PROCESS DESCRIPTION}

The industrial grinding process under consideration is a part of lead-zinc ore beneficiation plant. Ore beneficiation is carried out in mainly two stages: grinding and flotation. Pulverization of the ore to finely ground particles in wet grinding mills is performed in order to liberate the valuables, i.e. lead and zinc, from its associated gangue. The ground particles are then selectively floated in flotation cells for individual recovery of lead and zinc by various means of physical and chemical separation. The grinding circuit has three stages: 1) a rod mill in open circuit operation, 2) a ball mill in closed circuit operation, and 3) a two-stage classification unit (one hydro-cyclone for the primary classification and two hydro-cyclones in parallel for the secondary classification).

After crushing in primary and secondary crushers, the ore from the mine is sent to fine ore storage bin. Fresh ore feed from the fine ore storage bin along with water is fed to the rod mill. The rod mill discharge slurry is mixed with the ball mill discharge slurry in a sump known as the primary sump (sump-1). Water is added to the primary sump to reduce the pulp density to facilitate the flow of the slurry smoothly within the circuit. The slurry from the primary sump is fed to primary cyclone, the primary classification unit. The overflow from the primary cyclone goes to another sump, namely secondary sump, where water is added to lower pulp density further. The mixed slurry from the secondary sump is fed to the secondary cyclone, the secondary classification unit. The underflow product from both primary and secondary cyclones is fed to the ball mill. The overflow from the secondary cyclone is the final product from the grinding circuit and goes to flotation circuit as feed. The complete circuit configuration is given in Fig. 1. In this circuit, only the input and output streams are having the hardware sensors (shown by black circles in Fig. 1) that can indicate the status of key performance indicators (KPI) of the circuit (some properties of slurry at the final product stream) dynamically.

In the grinding circuit presented in Fig. 1, three main input process variables, which are also used typically as the manipulated variables for the control of grinding operation, are solid stream of raw ore and water streams going to primary and secondary sumps. The circuit has only one physical output that is the secondary cyclone overflow stream. The five KPIs identified for grinding circuit control are throughput (output 1), percentages of three size classes $(+150 \mu,-63 \mu$ and $-38 \mu)$ i.e. output 2 , output 3 , output 4 respectively and percent solids (output 5) present the final output stream, and the recirculation load (output-6). Here + sign is used to denote

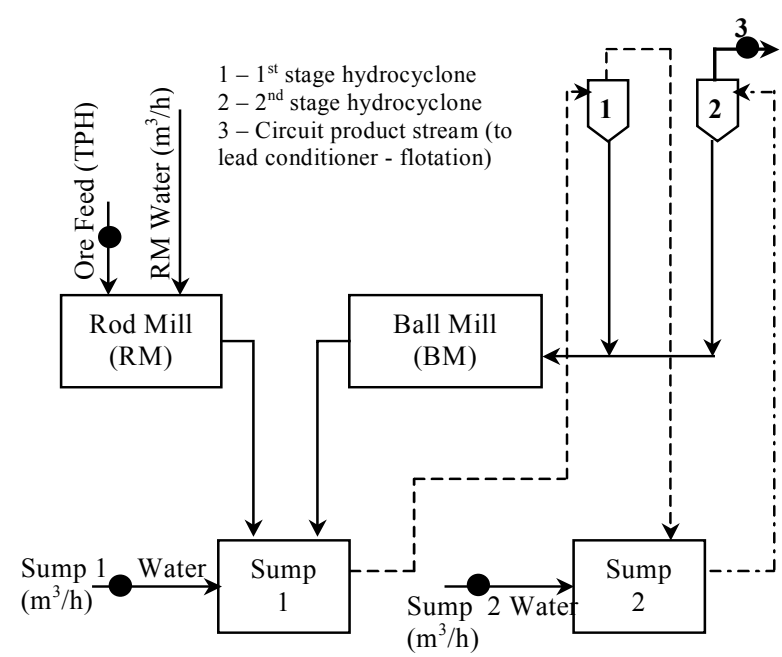

Fig. 1. Schematic of the industrial wet grinding process of lead-zinc ore beneficiation plant. 
percentage retained whereas - sign is used to denote percentage passing through the given mesh size in micron. These are termed as control variables and measured dynamically only at the output stream.

\subsection{Rigorous Validated Model for process emulation}

The hybrid (phenomenological and empirical) model of the above industrial grinding process is developed and validated with the plant data. Mathematically this hybrid model is a system of differential algebraic equations (DAEs) solved using the DASSL routines (Petzold, 1983). For this hybrid model, the details of the modelling and parameter estimation procedure, and results of the model validation with actual industry data are given in (Mitra and Gopinath, 2004). This hybrid model also includes as an output the recirculation load, an important KPI for the grinding operation, which is normally not measured online. This hybrid model represents the industrial plant operations very well across all possible operating zones and therefore can be considered as a close mimic of the plant. The simulator based on this rigorous hybrid model is used as a plant emulator in the current work. The simulator has 3 inputs and 6 outputs described in the previous section.

\section{PROPOSED MODELLING APPROACH}

The proposed approach of modelling the considered non-linear grinding process is based on the piece-wise linear approximation using multiple linear models. Hence, the over all operating region is divided into five operating zones defined by the second input i.e. a water flow rate to sump-1. Then, for each of the five operating zones, LTI MIMO models are identified. The non-linear process outputs are computed as a weighted sum of the outputs from each LTI MIMO model. The weights for each LTI model output is online determined using a special pseudo fuzzy logic supervisor. Fig. 2 shows the schematic diagram of the proposed modelling approach.

\subsection{Pseudo Fuzzy Supervisor}

The pseudo fuzzy supervisor is used for the smooth switching of the LTI MIMO model, mainly at the boundary of the different operating zones. As shown in Fig. 3, the weight computation logic of the pseudo fuzzy supervisor is similar to a typical trapezoidal fuzzy membership function (Driankov and Hellendoorn, 1993). The value of the membership function for each zone decides the weight of the linear model-output from the respective operating zone. When the current operating condition of the process exclusively falls within a particular operating zone, the weight of the LTI model corresponding to this zone is taken as 1. For all other model outputs, the weight is 0 . However, when the current operating condition of the process is at the boundary of two

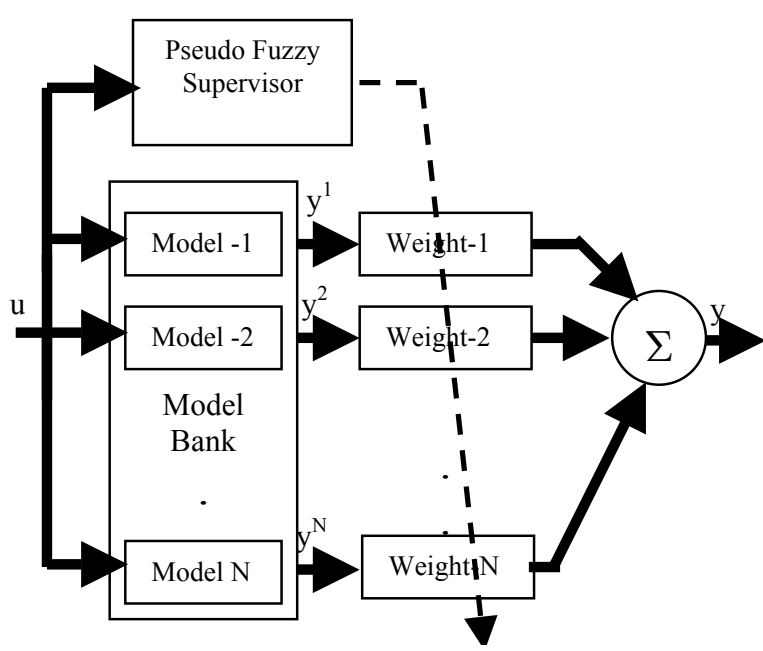

Fig. 2. Schematic of the proposed multiple linear model based piece-wise linearization approach for modelling the nonlinear zinc-ore grinding process

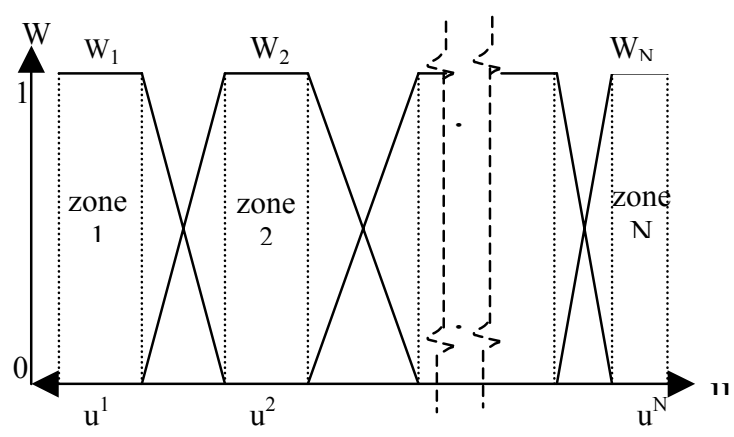

Fig. 3. Trapezoidal membership function schematic of the pseudo fuzzy supervisor defining weights of the LTI models

adjacent operating zones (e.g. zone-1 and zone-2), the weights for the outputs from the LTI models corresponding to these zones are computed as a real value within a real interval $[0,1]$. The values of the weights for models corresponding to these zones (e.g. zone 1 and zone-2) depend upon the vicinity of the current operating condition to a particular zone i.e. zone- 1 or zone- 2 , with the constraints of having sum of weights equal to 1 .

\subsection{LTI MIMO System Identification}

The proposed multiple linear model based non-linear modelling approach requires identification of the linear models. For which it is required to collect plant data across all operational regimes in which the grinding circuit is operated. For plant data collection, it is required to carry out well- designed plant tests covering various operating conditions and combinations of the process inputs (solids and water flow-rates). Performing such plant tests in all possible operating regimes is not an economically affordable task. Because, it requires disturbing the operational settings for running the plant in stable mode that incurs a huge loss for this energy intensive process. Besides, the data collection for system identification is a huge time consuming process. For 
these reasons, input-output data for the current work is not collected directly from the plant. Instead, the plant data is generated using the plant emulator described in section 2.1. After obtaining the data for each zone, the system identification step is required to find the linear models.

Several standard and popular linear system identification tools are available, e.g. System Identification Toolbox of MATLAB (Ljung, 1997), and can be used to identify LTI MIMO systems models using the input-output data from the system for different operating zones. However, in this work, recently proposed different approach of transfer matrix model identification (Barve and Junnuri, 2004) is used for linear system identification. In the proposed multivariable identification approach, MIMO system is represented using LTI transfer function (TF) matrix whose elements are the standard, fixed structure transfer functions like FOPDT, SOPDT, etc. These model structures are capable of well approximating very large class of systems found in practice. The system identification problem is then considered as the problem of simultaneously estimating the parameters of all TFs in the TF matrix. This is posed mathematically as the constrained optimisation problem, which minimizes some specific objective function based on the error between the actual and the linear model simulated response. A genetic algorithm is used to solve the proposed optimisation problem (Rao, 1996). The proposed identification approach has been successfully tested on several system identification benchmark data sets available at DaISy, (De Moor, 2004), (Barve and Junnuri, 2004). The DaISy provides benchmark data sets for various experimental and industrial systems covering wide range of application areas, such as chemical, electrical and mechanical systems.

\section{RESULTS AND DISCUSSION}

As described in the previous section, the first principle based, plant validated simulator is available for the considered process and is used to emulate or mimic the plant. This is preferred to performing tedious plant tests because a) the industrial plant tests affects the production during the plant test periods, hence they should be avoided, unless it is compulsory, and b) the plant emulator (mimic) that is validated earlier across the complete operating region by performing the plant tests is already available. Hence, in the sequel, measured or plant data means the data obtained from the rigorous model based plant emulator.

The considered multivariable grinding process has three input and six output variables. As described in section-2, the three input process variables are solid stream of raw-ore, water streams going to primary and secondary sumps. The output process variables are one physical output variable (i.e. secondary cyclone overflow stream), and five KPIs.
The five KPIs identified are throughput (output 1), percentages of three size classes $(+150 \mu,-63 \mu$ and $38 \mu$ ) i.e. output 2 , output 3 , output 4 respectively, percent solids (output 5) present in the final output stream, and the recirculation load (output-6).

First, the plant data is collected after applying the Pseudo Random Binary Sequence (PRBS) signal covering the complete operating region as the inputs. From the obtained plant data, it was observed that the considered wet grinding process shows the nonlinear behaviour. Particularly, the dynamic response of the second output (i.e. size fraction 150+) with respect to the first and third inputs (i.e. raw ore-feed and water flow rate to sump-2) change depending upon the current operating value of the second input i.e. the water flow rate to sump-1. Though, output 6 shows some non-linearity, it is not significant and is not considered as non-linear in this work. Thus, in the considered process output- 2 is considered to be non-linear, whereas the other five outputs are considered as linear.

For piece-wise linear approximation of the second output, the over all operating region is divided in five operating zones. The zones are defined based on the operating value of second input (i.e. a water flow rate to sump-1). The plant data is obtained for each of the five operating zone by applying the PRBS as inputs. However, in this case the first and third inputs cover the complete operating region, but the second input (i.e. the water flow rate to sump-1) is restricted to be within a particular operating zone. The LTI MIMO models are identified for each operating zone.

The outputs of the overall non-linear process are defined as a weighted sum of the outputs from each LTI MIMO model. The second input defines the operating zone of the non-linear process, it is considered as an input variable to the pseudo fuzzy logic supervisor. The five outputs from the fuzzy supervisor are weights for LTI models corresponding to each zone. After obtaining linear models for each zone, the proposed multi-model based non-linear model is simulated and validated in MATLAB/ SIMULINK (Mathworks, 2000). The identification and validation dataset of approx 5000 data-points are used in each zone for modelling the second output, whereas for modelling all other outputs, identification and validation datasets of 10000 and 50000 data-points are used. Finally, for the validation of overall non-linear model, a separate validation data set of 50000 data-points is used.

The performance of the identified models is analysed based on three well-known performance criteria (Ljung, 1999),(Chui and Maciejowski, 1998), (Barve and Junnuri, 2004) namely; mean square error (MSE), percentage simulation error (\% ERR), and cross-correlation coefficient (in percentage) between the simulated and an actual output (COR). As described above, five (multiple) models are considered for the second output. These performance 
Table 1: Performance measures for the multiple linear models identified using the transfer matrix approach of system identification

\begin{tabular}{llll}
\multicolumn{1}{l}{ Output } & MSE & AERR & COR \\
\hline Output-1 & 0.2613 & 13.85 & 99.04 \\
Output-2: Zone-1 & 0.0351 & 31.73 & 96.99 \\
Zone-2 & 0.0247 & 25.12 & 96.81 \\
Zone-3 & 0.0200 & 29.74 & 95.90 \\
Zone-4 & 0.0182 & 27.95 & 96.42 \\
Zone-5 & 0.0181 & 29.01 & 96.13 \\
Output-3 & 0.1600 & 12.51 & 99.25 \\
Output-4 & 0.1505 & 16.77 & 98.64 \\
Output-5 & 0.1512 & 14.68 & 98.79 \\
Output-6 & 1.2478 & 20.88 & 97.80 \\
& & & \\
\hline
\end{tabular}

Table-2: Comparison of the performance of the proposed multimodel based non-linear modelling approach and the single linear model approach

\begin{tabular}{llll} 
Approach & $\begin{array}{l}\text { Performance } \\
\text { Measure }\end{array}$ & Output-2 & Overall \\
\hline Single & MSE & 0.0824 & 1.6116 \\
Model & AERR & 86.92 & 28.38 \\
& COR & 94.45 & - \\
\hline Multi- & MSE & 0.0274 & 0.3330 \\
model & AERR & 28.9 & 18.07 \\
& COR & 96.31 & - \\
\hline
\end{tabular}

measures for the linear models for all outputs are shown in Table-1; see that performance measures for five zones are given for the second output.

In Table-2, for the second output, the performance measures of the proposed non-linear model is compared with the performance measures of the single linear model identified using data covering complete operating region (across all five zones). Though it may seem unnecessary to compare them expecting better results with non-linear models, the purpose of showing comparison is to find whether the improvement is significant or not. It is clearly observed that the significant improvement is possible by the proposed non-linear modelling approach. This justifies the exercise of modelling and using the proposed non-linear modelling approach for the considered wet grinding process.

It is observed that the performance of the proposed approach is superior justifying the use of multiple linear model based non-linear modelling. In Fig. 4 and 5 simulated and measured (i.e. rigorous plant emulator) response for all the six process outputs are shown. It can be seen that the response of the non-linear model obtained by the proposed approach matches well with the plant data (i.e. plant emulator data). Fig. 6 shows the comparison of the simulated responses for output- 2 obtained by a single overall linear model, and the proposed multiple linear models based non-linear simulation along with the plant data. It is clearly observed that the nonlinear model based on the proposed approach matches better compared to the single linear models.
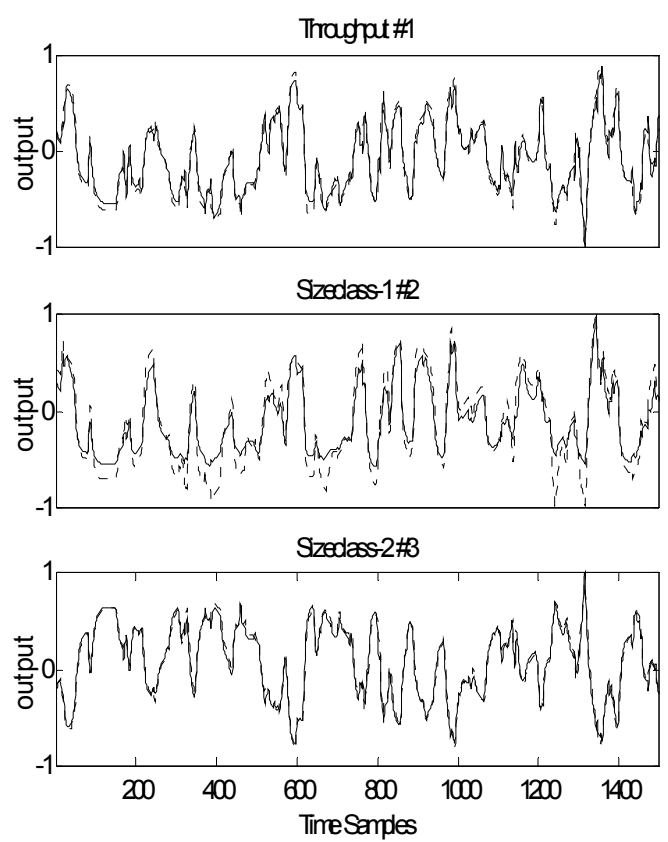

Fig. 4. Simulated response of the proposed non-linear model for outputs 1, 2 and 3: validation data (solid) simulated data (dotted)
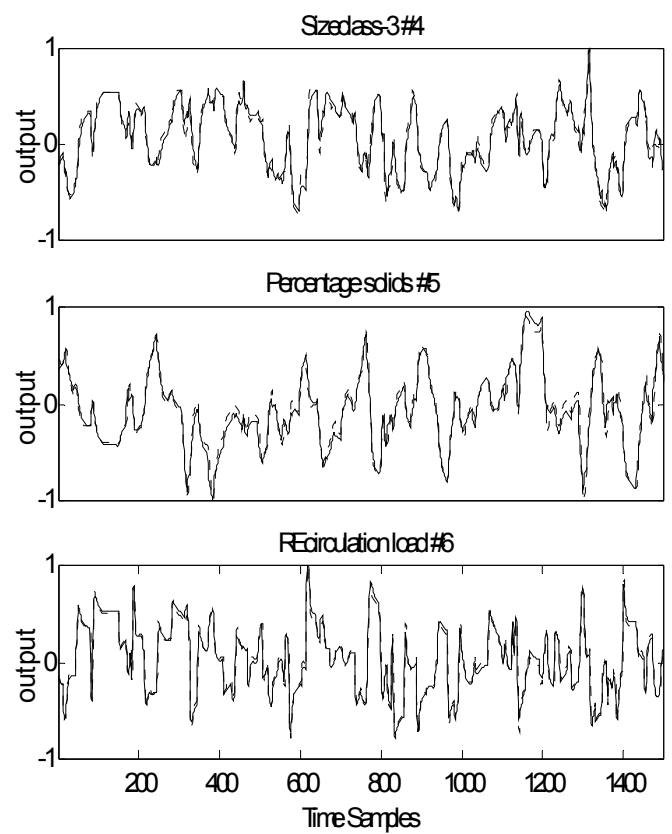

Fig. 5. Simulated response of the proposed non-linear model for outputs 4,5 and 6: validation data (solid) simulated data (dotted)

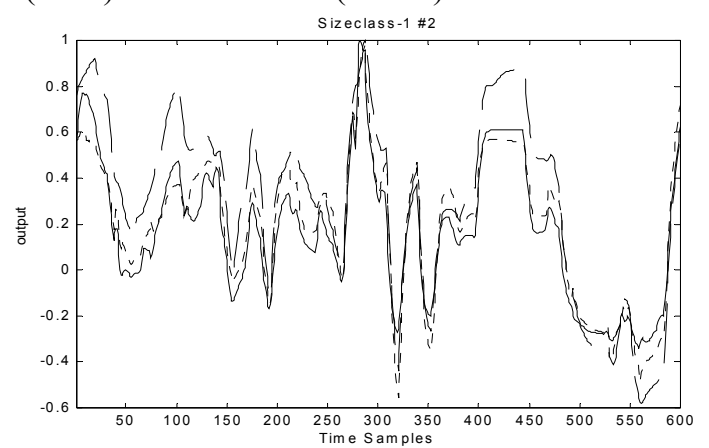

Fig. 6. Comparison of output-2 responses: Proposed non-linear model (dotted), Over-all single linear model (dashed) with the plant data (solid). 


\section{CONCLUSIONS}

The considered industrial wet grinding process can be modelled accurately using multiple linear models based non-linear modelling approach. The proposed approach shows significant improvement in the performance measures in comparison to the approach based on the single over-all linear model. Hence, for the considered process multiple linear model based NMPC should provide tighter control, compared to the single model based linear MPC. Though inherently non-linear modelling is possible for the same process using rigorous or other data based techniques, but they are not control-relevant from the model predictive control perspective. Because, their use in the industrial model predictive control is discouraged due to lack of reliable non-linear MPC algorithms based on inherently non-linear models. In this context, the proposed multiple linear model based non-linear modelling approach proves to be very useful and control-relevant, because it allows the use of reliable and proven linear model predictive control algorithms for the non-linear MPC control of the industrial wet grinding process.

\section{ACKNOWLEDGEMENT}

The authors acknowledge the support of Dr. Gautam Sardar, Head, Engineering Technology Development Group, and Dr. Ravi Gopinath, VP and Head, Engineering and Industrial Services, Tata Consultancy Services for their support without which this work could not have been possible.

\section{REFERENCES}

Barve J., and V.S.S.R. Junnuri (2004), System Identification using Transfer Function Matrix, Accepted for presentation in IEEE International Conference, RAM 2004, Dec. 2004, Singapore.

Birch, P. R. (1972),. An Introduction to the Control of Grinding Circuits Closed by Hydrocyclones, Minerals Sci. Engg., 4 (3), 55-66.

Bryson, A. E., Ho, Y. C., (1969). Applied Optimal Control, Blaisdell, Waltham, MA.

Chui, N. L. C., and J. Maciejowski (1998), Subspace Identification - A Markov Parameter Approach", Technical Report CUED/FINFENG/TR337/ Submitted to IEEE Trans. AC, Univ. of Cambridge, Cambridge.

De Moor B.L.R. (ed.) (2004) DaISy: Database for the Identification of systems, Department of Electrical Engineering, ESAT/SISTA, K. U. Leuven, Belgium, URL: "http:// www.esat.kuleuven.ac.be/sista/daisy/" June17, 2004.[Used datasets: robot_arm.dat.gz, dryer2.dat.gz]

Driankov D., Hellendoorn h. (1993), Introduction to Fuzzy Control, Springer-Verlag, NewYork.
Herbst, J. A., Fuerstenau, D. W. (1973). Mathematical Simulation of Dry Ball Milling Using Specific Power Information, Trans. AIME, 254, 343

Herbst, J. A., Rajamani, K. (1979). Evaluation of Optimising Control Strategies of Closed Circuit Grinding, In: Proceedings of the 13th International Mineral Processing Congress, Warsaw.

Herbst, J. A., Siddique, M., Rajamani, K., Sanchez, E. (1983). Population Based Approach to Ball Mill Scale-up: Bench and Pilot Scale Investigations, Trans AIME, 272, 1945-1954.

Kinneberg, D. J., Herbst, J. A. (1984). Comparison of Models for the Simulation of Open Circuit Ball Mill Grinding, Int. Jour. Of Min. Proc.

Lapidus, L., Luss, R., (1967). Optimal Control of Engineering Process, Blaisdell, Waltham, MA.

Ljung, L, (1997) System identification toolbox: User's guide. The Math Works Inc.

Ljung, L. (1999) System identification: Theory for the user. Englewood Cliffs, NJ: Prentice-Hall.

Lynch A. J., Rao, T. C. (1975). Modelling and Scale up of Hydro cyclone Classifiers, In: Proceedings of the 11th International Mineral Processing Congress, Cagliari, 245-269.

Maciejowski, J.M. (2002), Predictive Control with

Constraints, Prentice-Hall, London.

Math Works Inc. (2000), MATLAB User's guide v5.3, MA, USA.

Mitra, K., Gopinath, R. (2004). Multiobjective Optimization of an Industrial Grinding Operation Using Elitist Nondominated Sorting Genetic Algorithm, Chem. Engg. Sci., 59, 385396.

Morari, M., and J.H. Lee (1999) Model Predictive Control: Past, present and future, Computers and Chemical Engineering, 23, pp. $667-682$

Petzold, L. R., (1983). A description of DASSL: a differential/algebraic system solver. In: Stepleman, R. S. et al. (Eds.), Scientific Computing. North-Holland, Amsterdam, 65-68.

Rajamani, R. K., Herbst, J. A. (1984). Simultaneous Estimation of Selection and Breakage Functions from Batch and Continuous Grinding Data, Trans. Instn. Min. Metall., C93, C74-C85.

Rajamani, R. K., Herbst, J. A. (1991a). Optimal Control of a Ball Mill Grinding Circuit-I. Grinding Circuit Modeling and Dynamic Simulation, Chem. Eng. Sci., 46 (3), 861-870.

Rajamani, R. K., Herbst, J. A. (1991b). Optimal Control of a Ball Mill Grinding Circuit-II. Feedback and Optimal Control, Chem. Eng. Sci., 46 (3), 871-879.

Rao, S.S (1996), Engineering Optimization: Theory and Practice. NewYork: John Wiley.

Ramesh, R. Rao, B. Aufderheide, B. Wayne Bequette (2003), Experimental Studies on Multiple Model Predictive Control for Automated Regulation of Hemodynamic Variables, IEEE Trans. Biomedical Engineering, 50 (3), 277-288. 